\title{
XXXIII. On a kind of easily absorbed radiation produced by the impact of slowly moving cathode Rays; together with a theory of the negative glow, the dark space, and the positive column
}

\section{J. J. Thomson M.A. F.R.S.}

To cite this article: J. J. Thomson M.A. F.R.S. (1901) XXXIII. On a kind of easily absorbed radiation produced by the impact of slowly moving cathode Rays; together with a theory of the negative glow, the dark space, and the positive column , Philosophical Magazine Series 6, 1:4, 361-376, DOI: $10.1080 / 14786440109462624$

To link to this article: http://dx.doi.org/10.1080/14786440109462624

曲 Published online: 08 Jun 2010.

Submit your article to this journal ए

Џ Article views: 9

Q View related articles $\sqsubset$

4 Citing articles: 4 View citing articles 
T II E

LONDON EDINBURGH, AND DUBLIN

\title{
PHILOSOPHICAI MAGAZINE
}

\author{
AND \\ JOURNAL OF SCIENCE.
}

[SIXTH SERIES.]

APRIL 1901.

XXXIII. On a kind of easily Alsorbed Radiation produced l!! the Impuct of slowly moving Cathode Rays; together with a Theory of the Negative Glow, the Dark Space, and the Positive Column. By J. J. Тномson, M.A., F.R.S., Professor of Experimental Physics, Camloridge*.

THE following investigation originated in an attempt to find whether any radiation analogous to Röntgen radiation was produced at the surfaces of the anode or cathode-the places where the current through a rarelied gas passes from the gas to the metal.

For this purpose a tube like that shown in fig. 1 was

Fig. 1.

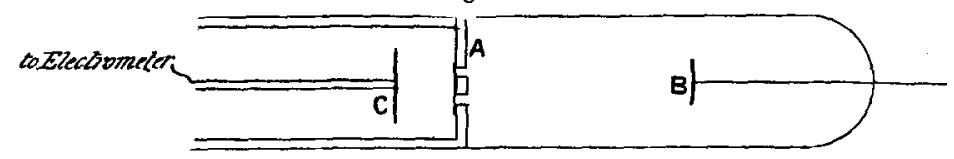

used. One end of the tube was made of a brass plate $A$, perforated with five holes placed near together ; the holes were covered on the outside by very thin aluminium foil, $\cdot 00043 \mathrm{~cm}$. thick. The face of the plate inside the tube was covered with a disk of mica, with a hole cut in the middle sufficiently large to allow the electric discharge to have arcess to the part of the plate containing the holes. A long metal tube was

* Comminicated by the Author.

Phil. Mag. S. 6. Vol. 1. No. 4. April 1901. 2 B 
fastened on the brass plate: inside this tube there was an insulated handle carrying the metal disk $\mathrm{C}$, which was connected with one pair of the quadrants of an electrometer. The metal tube and the brass end of the discharge-tube were kept permanently connected with the earth; the other electrode of the discharge-tube was an aluminium disk. To test whether any radiation passed through the windows in the brass plate the disk $C$ was charged up, and-the insulation having been found to be practically perfect when no discharge was passing through the tube-the discharge was turned on; it was then found that under certain circumstances the electricity leaked from $C$. This was the method most frequently used, but in other cases the presence of radiation was tested by taking photographs outside the tube behind the windows. In the earlier experiments, the discharge was produced by an induction-coil giving about $25 \mathrm{~cm}$. sparks, and it was found that even when the pressure in the tube was so high that the dark space was not more than 3 millimetres thick, the disk $\mathrm{C}$ began to leak perceptibly when the discharge passed through the tube. It leaked more quickly when the brass plate was the negative than when it was the positive electrode. The potential-difference between the electrodes was, however, less in the second case than in the first, as the mica in front of the brass plate made the effective area of this electrode less than that of the electrode $B$, and when the discharge passes between electrodes of different sizes the potential-difference between the electrodes is less when the larger electrode is cathode than when it is anode.

The rate of leak from the disk $\mathrm{C}$ was found to be independent of the sign of its electrification : and the disk did not receive a charge if it were uncharged to begin with. 'The leak shows that the gas outside the tube is ionized, and that this ionization is similar to that produced by Röntgen rays. To see whether the effects outside the tube corresponded in other respects to those produced by Röntgen rays, a photographic plate was placed outside the tube, behind the holes, and with an exposure of about 5 minutes very good photographs of the holes were obtained, the photographs being denser when the brass disk was cathode than when it was anode. There is thus outside the tube even when the gras is at a pressure very mnch greater than that in ordinary Röntgen tubes a radiation which possesses properties similar to the Röntgen radiation.

As the potential-difference required to produce the discharge at these high pressures is extremely small, it was thonght desirable to replace the induction-coil by a battery of small storage-cells. With these cells it was found possible 
Radiation produced by slowly moving Cathode Rays. 363

to get the radiation outside the tube; and as the results were much more regular than with the coil, and the current through the tube and the potential-difference between the electrodes much more easily measured, the coil was abandoned and the subsequent experiments made with the battery of cells.

The radiation obtained in this way is very easily absorbed, for if a screen of the very thin aluminium-foil used for the windows was placed between the windows and the disk, the rate of leak from the disk was reduced to about $1 / 6$ of its previous value. The radiation is also absorbed by a thin layer of air ; for when the tube containing the disk was exlausted and the rate of leak taken through gas at various pressures, it was found that the rate was not affected by the pressure until this fell below a certain value. This shows that it was not until this reduction of pressure took place that the radiation was able to reach the disk, which was about $1 \mathrm{~cm}$. from the window. The following numbers show the variation in the rate of leak with the pressure in the outside tube, the conditions in the discharge-tube being kept constant.

$\begin{array}{rc}\text { Pressure in Outer Tube. } & \text { Leak from lisk in one minute. } \\ 770 \text { millims. } & 87 \\ 270 \quad " & 90 \\ 100 \quad " & 64 \\ 45 \quad " & 37 \\ 10 \quad " & 11 \\ 5 \quad " & 3\end{array}$

Origin of the Radiation.-It was found that the radiation started at the place where the negative glow hit against a solid surface. Thus, when the window was cathode and the negative glow extended to the anode, the anode was the source of the rays. This was proved by deflecting the negative glow by a magnet. In this way the negative glow could gradually be displaced from the anode. As soon as the glow began to leave the anode the leak began to diminish, and when the glow was clear of the anode the leak ceased entirely. If the exhaustion of the tube was carried so far that the negative dark space reached to the anode, the rate of leak was very small : this point will be considered at more length later on.

After the source of the radiation had thus been determined, it seemed desirable to make experiments with a tube in which the window was not itself an electrode, and in which the position of the window in the negative glow could be varied. The tube (fig. 2) was constructed for this purpose. The 2 B 2 
metal tube, with the window, enclosing the charged disk $\mathrm{C}$ can be moved up and down by means of an indiarubber tube between the tube and the discharge-tube; $\mathrm{A}$ and $\mathrm{B}$ are aluminium disks used as electrodes. When $\mathrm{A}$ is made cathode, it is surrounded by a dark space which is followed by the luminous negative glow. The distance the glow extends up the vertical tube depends on the pressure of the gas and the magnitude of the electric current flowing through the tube. The current through the tube was adjusted by means of a liquid resistance, introduced in series with the tube. With

Fig. 2.

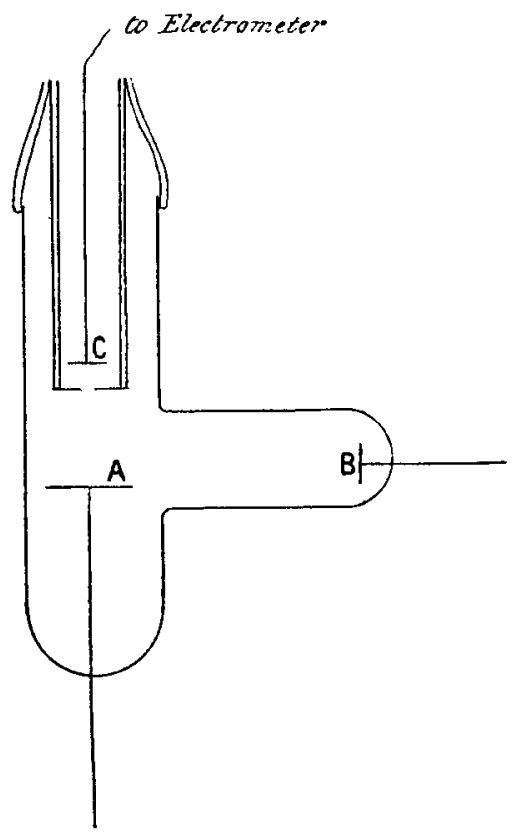

this adjustment very considerable variations were obtained in the length of the negative glow without altering the pressure of the gas. With this apparatus no leak occurred when the electrode A was positive; indeed, one could hardly be expected, as very little of the current went up the tube towards the window. When, however, A was made cathode, and a large enough current sent through the tube to make the negative glow reach the window, the charge leaked from the disk $\mathrm{C}$ behind the window. When the current was varied, the rate 
Radiation produced by slowly moving Cathode Rays. 365

of leak increased at one stage very rapidly with the current. With small currents there was hardly any leak; but as the current was gradually increased the rate of leak rapidly increased, at one stage much more rapidly than the current. I attribute this to the current when small taking almost exclusively the shortest path between the electrodes: when the current increases it spreads out and many more cathode-rays travel towards the perforated box.

Some interesting points were established by this apparatus. One was that the radiation which produced the leak was not due to the light coming from the luminous negative column, but arose from the impact against the windows of negatively clectrified particles travelling away from the cathode. This was proved by the effect of a magnet on the rate of leak: the negative glow follows the lines of magnetic force, so that when placed in a strong magnetic field, with the lines of force parallel to the face of the plate containing the windows, the luminous glow had a sharply-defined boundary parallel to the plate. The plate was then lowered down on to the top of this luminous negative glow ; there was, however, absolutely no leak from the disk, though, as soon as the magnet was taken away and the negative particles struck against the windows, there was a rapid leak. If the leak had been due to radiation coming from the luminous gas in the negative glow, the leak when the magnet was on would be greater than when it was off; for, in consequence of the concentration of the luminosity along the lines of magnetic force, the luminosity of the glow is increased by the magnetic field. Another very interesting point is that, although the rate of leak at first increases as the windows approach the cathode, yet when they get close enough to the cathode to enter the dark space, the rate of leak from the disk rapidly diminishes as the window moves nearer to the cathode, until when the window gets well within the dark space the leak practically ceases. The preceding experiment shows that it is not primarily due to the greater luminosity of the negative glow. To measure the way the rate of leak varied with the distance from the cathode, the arrangement was modified; since with the form of apparatus shown in fig. 2, the eathode-rays reaching the window form only a kind of bye-stream, the proportion which this bears to the main stream varying rapidly with the intensity of the current: it seems, therefore, desirable to place the tube containing the windows directly in the main stream. The arrangement adopted is shown in fig. 3 . The cathode was attached to a glass float, floating on the top of a mercury column; by altering the level of the mercury in the cistern, the cathode 
could be moved from one position to another. The tule with the aluminium windows was kept fixed between the fixed anode and the moveable cathode; when the cathode was moved the windows were thus brought into different positions in the negative glow. The potential-difference between the cathode and anode was measured by a Kelvin vertical voltmeter, the current through the tube by a D'Arsonval galvanometer; this current was adjusted by a liquid resistance

Fig. 8.

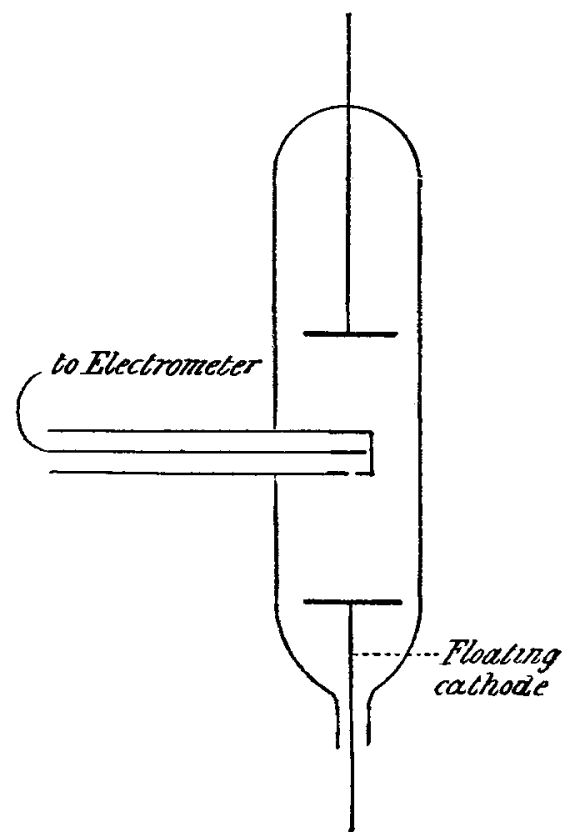

placed in series with the tube. The pressure in the tube was measured by keeping the tube connected with a mercurypump to which a McLeod gauge was attached. The following (p. 367) is a specimen of the observations made with this apparatus, showing the way in which the intensity of the radiation produced by the impact of the cathode particles: against the windows varies with the distance of the window: from the cathode.

The relation between the rate of leak and the distance from the window is shown graphically in fig. 4. The rate of 
Radiation produced by slowly moring Cathode Rays. 367

Pressure in Discharge-tube Size of Dark Space $4 \mathrm{~mm}$.

$\cdot 6 \mathrm{inm}$. of mercury.

\begin{tabular}{|c|c|c|c|}
\hline $\begin{array}{l}\text { Distance of } \\
\text { window from } \\
\text { surface of } \\
\text { cathode in } \\
\text { millimetres. }\end{array}$ & $\begin{array}{l}\text { Potential- } \\
\text { difference } \\
\text { between anorle } \\
\text { and cathode } \\
\text { in volts. }\end{array}$ & $\begin{array}{c}\text { Current through } \\
\text { tube in } \\
\text { nilliamperes. }\end{array}$ & $\begin{array}{l}\text { Rate of leak, } \\
\text { scale-divisions } \\
\text { per uninute. }\end{array}$ \\
\hline $\begin{array}{r}2 \\
4 \\
5 \\
10 \\
20 \\
30 \\
40\end{array}$ & $\begin{array}{l}1600 \\
1600 \\
1600 \\
1500 \\
1500 \\
1400 \\
1500\end{array}$ & $\begin{array}{l}8 \cdot 1 \\
93 \\
9 \cdot 3 \\
9 \cdot 2 \\
88 \\
8 \cdot 9 \\
88\end{array}$ & $\begin{array}{r}3 \\
20 \\
30 \\
23 \\
14 \\
8 \\
3\end{array}$ \\
\hline \multicolumn{2}{|c|}{$\begin{array}{l}\text { Pressure in Discharge-tube } \\
\quad \cdot 4 \mathrm{~mm} \text {. of mercury. }\end{array}$} & \multicolumn{2}{|c|}{ Dark Space $6 \mathrm{~mm}$. } \\
\hline $\begin{array}{r}3 \\
4 \\
5 \\
6 \\
8 \\
10 \\
20 \\
30 \\
40\end{array}$ & $\begin{array}{l}1700 \\
1700 \\
1700 \\
1700 \\
1700 \\
1700 \\
1700 \\
1700 \\
1700\end{array}$ & $\begin{array}{l}6.2 \\
6 \cdot 2 \\
70 \\
60 \\
66 \\
6 \cdot 6 \\
70 \\
70 \\
6.4\end{array}$ & $\begin{array}{r}21 \\
54 \\
105 \\
195 \\
150 \\
180 \\
66 \\
40 \\
25\end{array}$ \\
\hline
\end{tabular}

Fìg. 4.

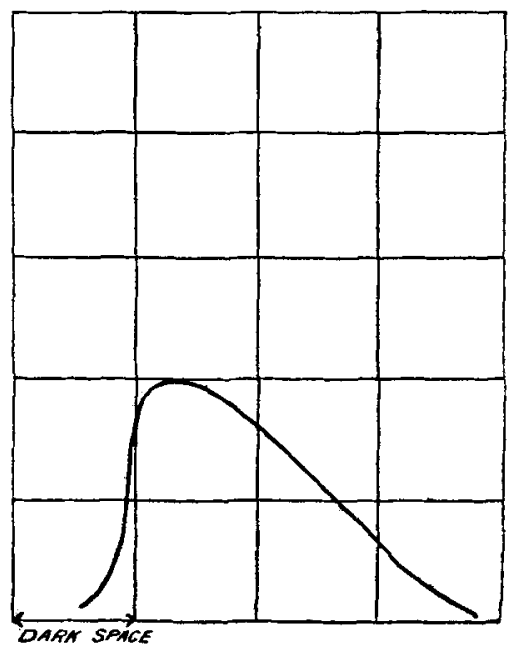


leak rises very rapidly to a maximum and then falls more slowly. It is apparent at a distance from the cathode which is a large multiple of the thickness of the dark space. The greater the current through the discharge-tube the greater is the rate of leak, and the further away from the cathode can it be detected. The greater the current, the greater is the extension of the negative glow.

As the radiation which produces the electricity from the disk shows all the characteristics of Röntgen radiation, although it has extraordinary small penetrating power, it seems legitimate to infer that it arises from the impact against the windows of the comparatively slowly moving corpuscles in the negative glow. The intensity of the radiation is a measure of the rate at which the energy due to these corpuscles is passing unit area at the place against which they strike. The numbers just given show that this rate is small inside the cathode dark space, rapidly increasing to a maximum at its luminous boundary, and then diminishing practically to zero at the positive end of the negative glow. This result is readily explained by the view I pat forward in a paper read before the Cambridge Philosophical Society in February 1900 (see Phil. Mag. 1. p. 278). On this view, the ionization which occurs when the electric discharge passes through a gas is due to the motion of other ions through the gas; thus each ion is, as it were, the child of another ion, the parent having been set in rapid motion by the electric field. The ionization by cathode and Lenard rays is a particular case of this principle. The view is further strengthened by the experiments recently made by Professor Townsend in the Cavendish Laboratory (Phil. Mag. Feb. 1901) on the ionization produced by the motion under an electric field through gases of low pressure of negative corpuscles produced by Röntgen rays : according to these experiments the negative corpuscle is a much more efficient ionizing agent in an electric field than the positive ion.

I shall now proceed to give a more detailed explanation of the phenomena in racuum-tubes from this point of view than I gave in my earlier paper.

Let us first consider the number of ions produced per second in unit volume of the gas. These ions are produced by the collision of the corpuscles with the molecules of the gas; and inasmuch as to ionize a molecule requires a finite amount of energy, if the energy of the corpuscles does not exceed a certain value, there will not be any ionization, while if the energy exceeds this critical value, then in a certain fraction of the collisions ionization will take place, this fraction increasing with the kinetic energy possessed by the particles. 
The mean kinetic energy of the corpuscles when there is no electric field is, on the assumption that the corpuscles behave like a gas, the same as the mean kinetic energy of the molecules of any gas at the same temperature. In an electric field, however, the corpuscles under the action of forces of the magnitude of those occurring in exhausted tubes, acquire velocities which are large compared with the average velocity when there is no electric field. Thus in these tubes the energy of the corpuscle is practically equal to that given to it by the field. Now if $X$ is the electric force, $\lambda$ the mean free path of a corpuscle, the mean kinetic energy acquired by the corpuscle in the electric field will be proportional to $\mathrm{X} e \lambda$, the work done on it by the electric field during its free path; $e$ is the charge of electricity on the corpuscle. A certain fraction of the whole number of collisions will lead to ionization, the value of the fraction depending upon the kinetic energy of the corpuscles; we shall denote the value of this fraction by $f(\mathrm{X} e \lambda)$, where at present all we know about $f$ is that it cannot exceed unity however great $\mathrm{X} e \lambda$ may be, and that it vanishes when $\mathrm{X} e \lambda$ falls below a certain limiting value.

If $u$ is the average velocity of a corpusele, $\lambda / u$ is the interval between two collisions; hence each corpuscle makes $u / \lambda$ collisions in unit time, so that if $n$ is the number of corpuscles in unit volume, $m u / \lambda$ is the total number of collisions in unit volume per unit time, the number of ions produced in unit volume per second is therefore equal to $\frac{n u}{\lambda} f(\mathrm{X} e \lambda)$.

The corpuscles ultimately get attached to molecules, thereby losing their mobility and, to a great extent at any rate, their power of ionizing other molecules. We shall suppose that in a certain fraction of the number of . collisions the effect of the collision is to leave the corpuscle sticking to the molecule with which it collided, and thus to end its career as an ionizing agent.

Let $\beta$ be the fraction of the whole number of collisions where this occurs, then the rate at which the corpuscles disappear is per unit volume equal to $\beta n u / \lambda$. The rate at which the ions are increasing per unit volume is therefore equal to

$$
\frac{m u}{\lambda}\{f(\mathrm{X} e \lambda)-\beta\} \text {. }
$$

If we consider the case of a tube in which the ions are moving parallel to the axis of $x$, then we have by the equation of continuity,

$$
\frac{d n}{d t}+\frac{d}{d x}(n u)=\frac{n u}{\lambda}\{f(\mathrm{X} e \lambda)-\beta\},
$$


where $x$ is measured from the cathode to the anode. When the discharge is steady $d n / d l=0$, and the equation becomes

$$
\frac{d}{d} \cdot(m u)=\frac{n u}{\lambda}\{f(\mathrm{X} e \lambda)-\beta\} \ldots . .
$$

Integrating this equation, we find

$$
\log n u=\mathrm{C}+\int_{\lambda}^{2} \frac{1}{\lambda}\{(\mathrm{X} e \lambda)-\beta\} d x
$$

where $\mathrm{C}$ is the constant of integration. From this equation we can find $n n$ when $f$ and $\beta$, and the distribution of electric force along the tube, are known.

In any region of the tubs where the conditions do not cbange as we travel along the line of discharge, $d(n u) / d x=0$; hence from equation (1) in this region $f(\mathrm{X} e \lambda)-\beta=0$, i. e. $\mathrm{X} \lambda$ has a definite value determined by this equation; as neither $\beta$ nor $f$ involves the current through the gas or the pressure of the gas in the tube, $X \lambda$ does not depend upon the current or the pressure: thus $X$ will vary inversely as $\lambda$. As $\lambda$ the mean free path of the corpuscles is inversely proportional to the pressure of the gas, it follows that in a uniform part of the discharge $\mathrm{X}$ must be proportional to the pressure. We get this uniformity along the line of discharge in the case of the uniform positive column in the discharge at low pressures, and also in the case of long sparks at higher pressures. The following results are given by Skinner (Phil. Mag. Dec. 1900) for the gradient along the positive column at various pressures $x^{3}$ for the discharge through nitrogen.

$$
\begin{array}{ccc}
p . & \mathrm{X} . & \mathrm{X}_{/ p} . \\
.6 \mathrm{~mm} . & 27 \text { volts } / \mathrm{cm} . & 45 \\
1.0 \mathrm{~mm} . & 40 \text { volts } / \mathrm{cm} . & 40 \\
1.5 \mathrm{~mm} . & 56 \text { volts } / \mathrm{cm} . & 38
\end{array}
$$

While for sparks in air Liebig found :-

$$
760 \mathrm{~mm} \text {. } \quad 31,000 \text { volts } / \mathrm{cm} . \quad 40 \cdot 8
$$

Thus through a very wide range of pressures there is but little change in the rnlues of $\mathrm{X} / \mathrm{p}$. The value of $\mathrm{X}$ in the uniform positive column is the minimum strength of the field which can increase the number of ions in a gas at the same pressure by setting in motion previously existing ions. If wo produce ions in a gas by Röntgen rays, the current through the gas will not exceed its saturation value until the electric field attains this strength.

The condition $f(\mathrm{X} e \lambda)-\beta=0$ expresses the condition that ench corpuscle produces one and only one other corpuscle 
Radiation produced by slowly moving Cathode Rays. 371 before it disappears. The condition $\mathrm{X} \lambda=\mathrm{a}$ constant implies that whenever a uniform discharge is taking place, the kinetic energy possessed by the corpuscles has a definite value independent of the pressure of the gas or the current through it.

The differential equation giving the distribution of electric force in the discharge-tube when the discharge does not vary with the time may be found as follows. If $i$ is the current through the tube, $m$ the number of positive ions per unit volume, $v$ the velocity of these ions, then

$$
n u+m v=i \text {. }
$$

As the kinetic energy possessed by the ions is proportional to $X \lambda$, we may put

$$
u=k_{1} \sqrt{\mathrm{X}}, \quad v=k_{2} \sqrt{\mathrm{X}},
$$

where $k_{1}$ and $k_{2}$ are constants depending on the density of the gas. Substituting these values, we get

$$
n k_{1}+m k_{2}=\frac{i}{\sqrt{X}} \cdot \text {. . . . . }
$$

Now

$$
\frac{d \mathrm{X}}{a_{c} t}=-4 \pi \rho \text {, }
$$

where $\rho$ is the density of the electrification, and $\rho=(m-n) \epsilon$; the minus sign occurs in this equation because we have taken as the positive direction of $x$ that from the cathode to the anode, while $\mathrm{X}$ is measured in the opposite direction; thus

$$
n-m=\frac{1}{4 \pi e} \frac{d \mathrm{X}}{d x} ; . . . . . .
$$

hence from (2) and (3)

or

$$
n\left(k_{\mathrm{f}}+k_{2}\right)=\frac{i}{\sqrt{\mathrm{X}}}+\frac{k_{2}}{4 \pi e} \frac{d \mathrm{X}}{d x}
$$

$$
n \imath=\frac{k_{1}}{k_{1}+k_{2}} i+\frac{k_{1} k_{2} v \overline{\mathrm{X}}}{4 \pi e\left(k_{1}+k_{2}\right)} \frac{d \mathrm{X}}{d x} \cdot . .
$$

Hence from equation (1) we have

$$
\begin{aligned}
\frac{k_{1} k_{2}}{4 \pi e} \frac{2}{3} \frac{d^{2} \mathrm{X}^{\frac{3}{2}}}{d x^{2}} & =\frac{n u}{\lambda}\{f(\mathrm{X} e \lambda)-\beta\} \\
& =\left(\frac{k_{1}}{k_{1}+k_{2}} \frac{i}{\lambda}+\frac{2}{3} \frac{k_{1} k_{2}}{4 \pi e \lambda\left(\bar{k}_{1}+k_{2}\right)} \frac{d \mathrm{X} \frac{3}{2}}{d x}\right)\{f(\mathrm{X} e \lambda)-\beta\},
\end{aligned}
$$

a differential equation to determine $X$.

We see from this equation that when $\{f(X e \lambda)-\beta\}$ is 
positive, $i$. e. when $\mathrm{X}$ is greater than $\mathrm{X}_{0}$, the value it has in the uniform positive column $\frac{d^{2} \mathrm{X}^{3}}{d x^{2}}$ is positive, i. e. the graph of $\mathrm{X}^{\frac{3}{2}}$ is convex to the axis of $x$, while when $\mathrm{X}$ is less than $\mathrm{X}_{0}$ it is concave to that axis.

Though the differential equation which gives $\mathrm{X}$ is a somewhat complicated one, yet we can without much difficulty see what must be the general nature of the distribution of electric force along the tube. We can do this from the principle that the graph for $\mathrm{X}^{\frac{3}{2}}$ must be convex or concave to the axis of $\mathrm{X}$ according as $\mathrm{X}$ is greater or less than $\mathrm{X}_{0}$; and that the electric force is increasing or diminishing as we move towards the anode, according as there is an excess of negative or positive electrification at the place of observation. Suppose, then, that we start with a few negatively electrified corpuscles near the cathode; under the strong field near the cathode these will acquire sufficient energy to ionize the gas, fresh ions will be.produced; and since the negative ones are driven away by the electric field, there will be an excess of positive ions next the cathode, and thus the electric force will diminish as we leave the cathode. The graph for $X_{2}^{\frac{\pi}{2}}$ (fig. 5) will be convex to $x$ until we come to $\mathrm{P}$, the place

Fig. 5.

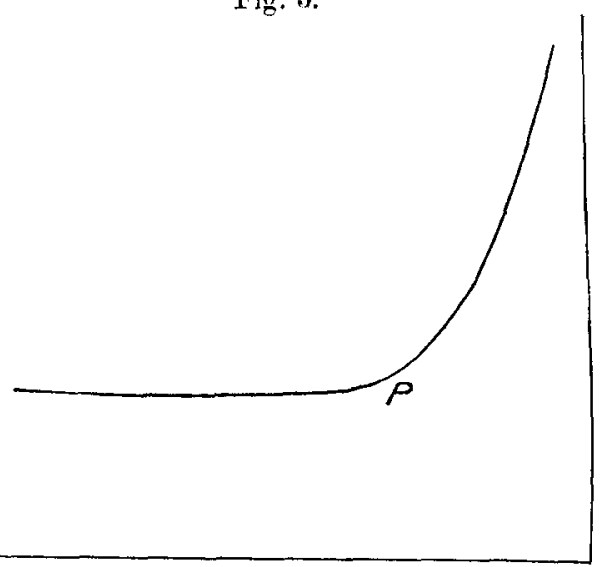

where $\mathrm{X}$ satisfies the equation $f(\mathrm{X} e \lambda)-\beta=0$, i.e. until $\mathrm{X}=\mathrm{X}_{0}$; at $\mathrm{P}$ the curvature changes, and there are two cases to be considered. In the first case the tangent to the graph is horizontal, in this case the rest of the graph will be the horizontal line through $\mathrm{P}$ : this straight part of the curve corresponds to the uniform positive column, and the case is 
Radiation produced by slowly moving Cathode Rays. 373

that of a uniform positive column reaching right up to the negative glow with no Faraday dark space between them. The second case (fig. 6) is when the tangent at $\mathrm{P}$ is not

Fig. 6.

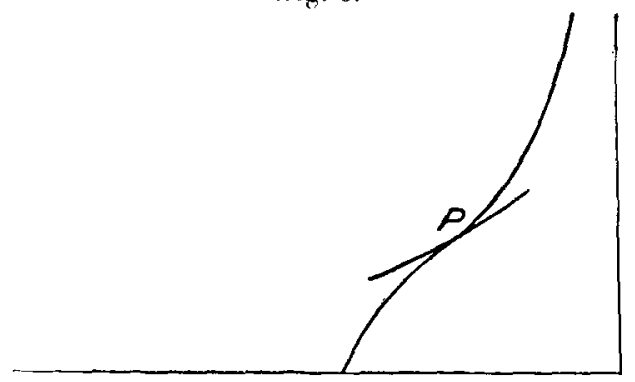

horizontal : here the curve must cross the tangent at $P$, becoming concave to $x$, and ultimately reaching the axis. Our equations, however, are not true when $\mathrm{X}$ is very small, is we have issumed that the field is strong enongh to cause the kinetic energy given to the corpuseles by the field to be large compared with the energy they would possess at the same temperature if not acted on by the electric force; we are not therefore at liberty to follow the curve into the region of very small electric forces.

The effects near the cathode are probably in practice complicated by the action of the 'Entladungstrahlen,' discovered by $\mathrm{E}$. Wiedemann, which are given out by the negative glow and, indeed, by all luminous parts of the discharge. I have shown that these rays possess the power of ionizing a gas through which they pass. These rays would therefore increase the ionization in the gas, and would keep the graph for $\mathrm{X}^{\frac{3}{2}}$ convex to the axis even though the force falls below the value of $X_{0}$ : we might thus, under the influence of these rays, get a graph such as that in fig. 7, and this seems to resemble more closely than fig. 6 the distribution of electric force, observed by Graham (Wied. Ann. lxiv. p. 49) in the neighbourhood of the cathode. The intensity of these rays will diminish as we recede from the cathode, and we shall sooner or later reach a place Q, fig. 7, where the ionization exceeds the recombinations; here the graph becomes concave to the axis and continues so until $\mathrm{X}$ again reaches the value $X_{0}$ at $R$. Here again there are two cases to be considered: (1) when the tangent at $R$ is horizontal; in this case the rest of the graph is the horizontal straight line through $R$, and the case is that of a uniform positive column separated by a Faraday dark space from the negative glow. 
The other case is when the tangent at $R$ is not horizontal: here the curve crosses the tangent, becoming convex to the

Fig. 7.

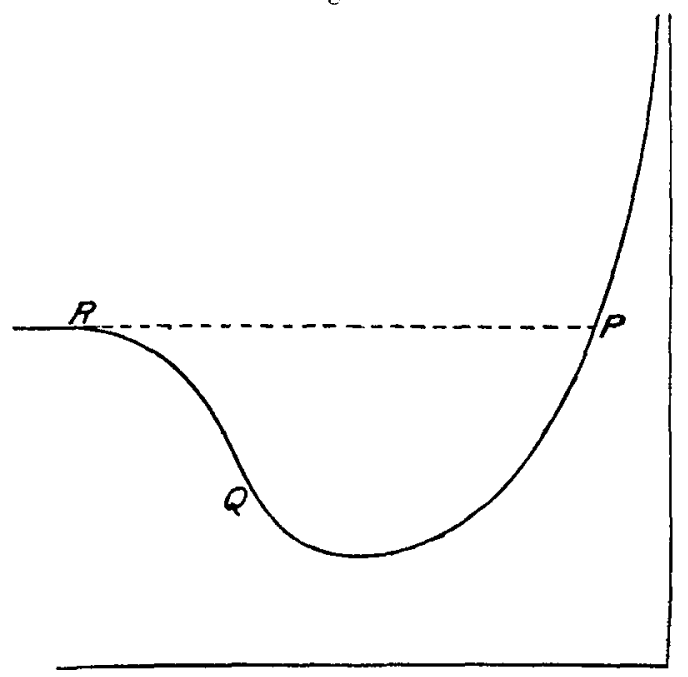

axis, and $\mathrm{X}$ continually increases as we approach the anode. A1ter passing $\mathrm{R}, f(\mathrm{X} e \lambda)-\beta$ is always positive, and hence by equation (1) $n u$ continually increases, in more than geometrical progression, as we travel towards the anode. Now, when the discharge is steady, $n u+m v=i$, hence $n u$ cannot exceed $i$. Hence in the case when the graph rises through $\mathbf{R}$ there must ultimately be instability, or rather unsteadiness; this will occur when the force reaches a value such that $n u=i$, or by equation (4) when

$$
\frac{4 \pi e}{k_{1}} i=\frac{2 d \mathrm{X}^{3}}{3}-\frac{1}{d x}
$$

that is when the tangent to the graph of $\mathrm{X}^{3}$ makes a certiin angle with the axis of $x$. For values of $\mathrm{X}$ greater than this $n u$ would be greater than $i$, and the current would no longer be steady. Let $\mathrm{S}$, fig. 8 , be the point where $n u=i$, then since negative electricity is streaming out from the region between $S$ and the cathode faster than it enters it, there will soon be an excess of positive electricity behind $\mathrm{S}$ : this will lower the electric force between $S$ and the anode, so that the graph for $X$ past $S$ will be somewhat as in fig. 8 (this represents the average force, as from the preceding 
Radiation produced by slowly moving Cathode Rays. 375

reasoning it must vary somewhat with the time); this branch of the curve can only extend to $\mathrm{S}^{\prime}$, where again

$$
\frac{4 \pi e}{k_{1}} i=\frac{2 d^{3} \mathrm{X}^{\frac{3}{3}}}{3} \frac{1}{d x} ;
$$

it will be succeeded by other similar branches. Thus we should get the mean electric force in the positive column represented by a fluctuating curve; this, as Graham and Wilson have shown, is the case where the positive column is striated.

Negative Dark Space.-The energy communicated by the corpuscles to the gas through which they move will be proportional to the product of the number of collisions between the corpuscles and the molecules and the kinetic energy possessed by a corpuscle. Now we have seen that the number of collisions is $n u / \lambda$, while the energy possessed by the corpuscles is proportional to $X \lambda$. Thus the energy given to the molecules by the corpuscles is proportional to $n . u \mathrm{X}$. Now, on the view we have taken, $n$ is very small close to the cathode, while $u \mathrm{X}$ is very small in the region about $\mathrm{Q}$ (fig. 8). Thus

Fig. 8.

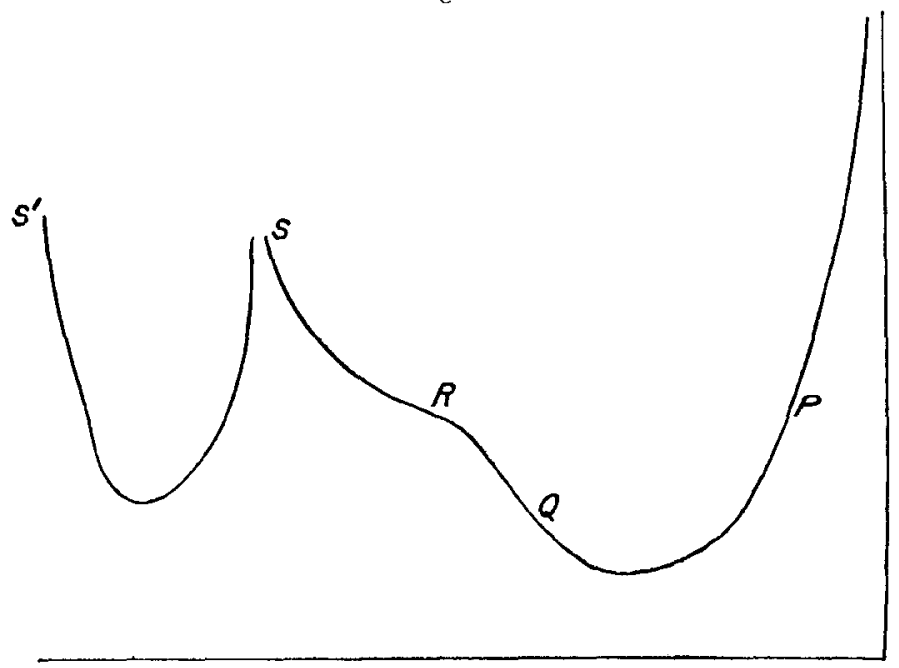

this product would be small close to the cathode, and also outside the negative glow; there would thus be some place between the two where the product is a maximum. Thus, if we take the luminosity of the glow as a measure of the energy 
376 Radiation produced by slowly moving Cathode Rays.

communicated by the corpuscles to the molecules of the gats, the luminosity would be small near the cathode (hence the dark space), would reach a maximum (at the luminous boundary of the dark space), and then diminish through the negative glow. The darkness near the cathode would be exaggerated if the pressure around the cathode were less than that in the rest of the tube. I think there is some evidence that this is the case, and hope to return to this point on a future occasion.

Fall of Potential near the Anode.-When we have a uniform positive column, the electric force along it is constant, and hence there are as many positive as negative ions in this region; hence the number of positive ions which cross the tube, going from anode towards cathode in unit time, is

$$
\frac{k_{i}}{k_{1}+k_{2}} i \text {. }
$$

The ions which cross a section at $\mathrm{P}$ have to be produced between $\mathrm{P}$ and the anode. Now, if these ions are produced by the action of the moving corpuscles, the number of ions produced in unit time is

$$
\int \frac{n u}{\lambda} f(\mathrm{X} e \lambda) d x
$$

where the integration extends over the space between the anode and $\mathrm{P}$, hence

$$
\int \frac{m u}{\lambda} f(\mathrm{X} e \lambda) d u \leftarrow \frac{k_{2}}{k_{1}+k_{2}} i .
$$

Now $m$ is not greater than $i$, hence

$$
\int \frac{1}{\lambda} f(\mathrm{X} e \lambda) d x \nLeftarrow \frac{k_{2}}{k_{1}+k_{2}} .
$$

Suppose, for example, $f(\mathrm{X} e \lambda)=\mathrm{AX} e \lambda$, where $\mathrm{A}$ is a constant, then the equation becomes

$$
\mathrm{A} e \mathrm{~V} \leftarrow \frac{k_{2}}{k_{1}+k_{2}}
$$

where $V$ is the difference of potential between the anode and $P$. Hence we see that there must he a finite difference of potential between the anode and the nearest point where the electric force in the positive column is constant. Such a fall of potential at the anode has been observed by Skinner; and we hope to return to the comparison of the results of this theory with the results of Skinner's experiments, as well as the explanation in a similar way of the fall of potential at the cathode, in a future paper. 